\title{
Immunocytochemistry and DNA-image cytometry in diagnostic effusion cytology I. Prevalence of markers in tumour cell positive and negative smears
}

\author{
Helma Motherby a,*,**, Mary Kube a, \\ Nikolaus Friedrichs ${ }^{\text {a }}$, Bahram Nadjari ${ }^{\text {a }}$, \\ Kristiane Knops ${ }^{\mathrm{a}}$, Andreas Donner ${ }^{\mathrm{b}}$, \\ Betty Baschiera ${ }^{\mathrm{c}}$, Peter Dalquen ${ }^{\mathrm{c}}$ and \\ Alfred Böcking ${ }^{\mathrm{a}}$ \\ ${ }^{\mathrm{a}}$ Institute of Cytopathology, Heinrich Heine \\ University, Moorenstr. 5, D-40225 Düsseldorf, \\ Germany \\ ${ }^{\mathrm{b}}$ Institute of Pathology, Heinrich Heine University, \\ Moorenstr. 5, D-40225 Düsseldorf, Germany \\ ${ }^{\mathrm{c}}$ Division of Cytopathology, Institute of Pathology, \\ University Hospital Basel, Schönbeinstr. 40, \\ CH-4003 Basel, Switzerland
}

Received 18 February 1999

Accepted 10 June 1999

To determine the prevalence of immunocytochemical positivities for a panel of antibodies in benign and malignant cells in effusions with known follow-up in order to use these as diagnostic markers. Besides their ability to identify malignant epithelial cells their contribution to the differential diagnosis between carcinomatoses and mesotheliomas was investigated.

\footnotetext{
${ }^{*}$ Correspondence to: Dr Helma Motherby, Tel.: +49 211 8118346; Fax: +49 2118118402

** Dr Motherby is Assistant Medical Director, Institute of $\mathrm{Cy}$ topathology; Mrs Kube is Biological Technical Assistant, Institute of Cytopathology; Mr Friedrichs is Medical Student, Institute of Cytopathology; Dr Nadjari is Resident, formerly Institute of Cytopathology, now Institute of Pathology, Charite, Humboldt University of Berlin; Mrs Knops is Cytotechnician, Institute of Cytopathology; Dr Donner is Pathologist, Institute of Pathology; Mrs Baschiera is Cytotechnician, Division of Cytopathology, Institute of Pathology, University Hospital of Basel, Switzerland; Dr Dalquen is Prof. of Pathology and Head of the Division of Cytopathology, Institute of Pathology, University Hospital of Basel, Switzerland; Dr Böcking is Professor of Pathology and Head of the Institute of Cytopathology.
}

101 tumour cell positive and 53 negative effusions were stained with 12 different antibodies. Results were scored semiquantitatively per cell type. Furthermore, DNA-image cytometry was performed.

While prevalence of Ber-EP4 positivity was $95.4 \%$ in metastatic carcinoma cells, it was $0 \%$ in those from mesotheliomas. No cell type reacted with this marker in benign effusions $(0 \%)$. Ber-EP4 correctly differentiated between metastatic carcinoma and mesothelioma in $98.0 \%$. Prevalence of DNA-aneuploidy was $95.4 \%$ in metastatic carcinomas, $57.1 \%$ in mesotheliomas and $0 \%$ in reactive effusions.

Combining immunocytochemistry (Ber-EP4 positivity) and DNA-image cytometry (aneuploidy) results in a $100 \%$ detection of metastatic carcinomatoses and $57.1 \%$ of mesotheliomas. Both markers furthermore allowed a correct differentiation of these entities in $98 \%$.

\section{Introduction}

Sensitivity of conventional cytology for the detection of malignant cells in effusions is unsatisfactory, about $58 \%$, specificity is about $97 \%$ (average from a literature review of 6,001 cases from 6 studies [66]). This diagnostic uncertainty manifests also in diagnostically "equivocal" effusions showing "inconclusive cells" or "cells suspicious for malignancy". Improvement of diagnostic accuracy is therefore necessary in effusion cytology. Not so much the largest possible number of metastatic cancers correctly diagnosed, but the smallest number of carcinomatoses incorrectly diagnosed is important [42]. Thus, sensitivity should be improved without lowering specificity.

The diagnostic value of immunocytochemistry for the identification of malignant cells in effusions has often been emphasized $[7,8,37,50,62,63]$. There is a great number of publications on immunohistochemical markers distinguishing between primary (mesothe- 
liomas) and secondary tumours (metastatic carcinomas) of the serous membranes on paraffin embedded histologic material [24,25,32,45,49,54,55,59,61]. On the other hand there is a number of publications on the use of specific antibodies for the immunocytochemical identification of primary sites of different metastatic carcinomas in effusions, e.g., of the ovary [20,35], the breast [34] or both [5], or of bronchial carcinoma [36] in effusions. In our study of the literature we have considered only those publications in which antibodies were applied without restriction to a specific primary tumour. 41 publications $[1-4,6,10,17-19,22,23,26,28-33,39-41,43,44$, 46-48,50-53,58,60,62-65,67-71] were found in which prevalences of markers for malignant and benign cells in effusions were recorded. The data for the ten most commonly applied antibodies were compared. 17 of these 41 studies $[3,19,23,26,28,33,41,43,44,48,50,58$, $60,62,65,67,69$ ] differentiate between effusions due to metastatic carcinomas and those due to mesotheliomas. Although 12 of these publications [2,10,22,23, $29,39-41,46,51,64,68]$ have also studied cytodiagnostically equivocal effusions only two of them, Illingworth et al. [39] and Matter-Walstra and Kraft [51] have calculated sensitivity, specificity, positive or negative predictive values of markers to identify malignant cells, but without differentiating between those due to metastatic carcinomatoses and those due to malignant mesotheliomas. Eight further authors [6,26,31,41,48, $53,62,70]$ have reported on "sensitivity", "specificity", "positive and negative predictive values", but as they have not included equivocal effusions in their diagnostic categories, to our opinion they have incorrectly applied these definitions. Eight other authors [17,26,29, $31,33,46,50,62]$ suggest the combined use of different immunocytochemical markers ("panels") for diagnostic purposes.

The extreme differences in reported prevalences of marker positivities in different cell types seem, apart from influences by different patient populations, to be due to differences in methodology. This makes data difficult to compare:

Studies used different fixations: air-drying, acetone, methanol, acetone plus methanol, ethanol, Carnoy's solution, Bouin's fixative, formalin, paraformaldehyd and formol sublimate. Furthermore, the investigations differ in techniques of preparation: cell blocks (paraffin-histology), cytospins or sediment smears (cytology). Yet, both fixation and cell preparation largely influence stabilization and presentation of antigen epitopes [9]. Furthermore, a wide variety of staining methods was applied, which accounts for differences in specificity and amplification of the reaction [9] the indirect peroxidase system (IP), peroxidase-anti-peroxidase-complex system (PAP), alkaline phosphatase-anti-alkaline-phosphatase-complex system (APAAP), (strept-)avidin-biotincomplex system ((strept-)ABC). The antibodies chosen by different authors vary strongly in their potential to detect epithelial cells and in their ability to differentiate these from benign or malignant cells of mesothelial origin: anti-AUA-1, -BCA-225, Ber-EP4, -B72.3, -calretinin, -CA1/2, -CEA, -cytokeratins, -83kD, -desmin, -EMA, -HEA 125, -HBME-1, -HMFG-2, -LCA, -Leu-M1, -MOC-31, -p53, -S-100 protein, -vimentin. The number of cases investigated differs from 25 to 227 (average 100.5). In several studies it is not obvious whether immunocytochemical results were compared with conventional cytologic diagnoses, with clinic or histologic followup.

In this study we applied twelve different antibodies against the following epitopes: Ber-EP4 (surface and cytoplasmic glycoprotein), Lu5 and MNF 116 (cytoplasmic cytokeratins), EMA (epithelial membrane antigen, a human milk fat globulin), B72.3 (cytoplasmic tumour-associated glycoprotein), Leu-M1 (cytoplasmic oligosaccharid), CEA (cytoplasmic carcinoembryonic antigen, a heavily glycosilated protein), CA-125 (membrane highmolecular mucin), HBME-1 (cytoplasmic mesenchymal marker), vimentin and desmin (cytoplasmic intermediate filaments) and MAC 387 (cytoplasmic myeloid-histiocyte). The choice of these antibodies was made, as on the one hand the most commonly applied antibodies (e.g., anti-CEA, -EMA, -cytokeratins), on the other hand those described as the most sensitive ones to detect epithelial cells and the most specific ones to differentiate between epithelial and mesothelial cells (e.g., Ber-EP4, -Leu-M1). Only recently we routinely apply the antibody anticalretinin, a mesothelial marker [6,27].

The diagnostic value of DNA-cytometry for the identification of malignant cells in effusions has been demonstrated by our group in two previous studies [56, 57].

The aim of the present study was to investigate the prevalence of cellular positivity for different antibodies in tumour cell negative, and positive effusions and to consider the usefulness of their combination as a panel. In parallel, the prevalence of DNA-aneuploidy in malignant and benign cells of effusions was determined in order to analyze the ability of both adjuvant methods to increase the diagnostic accuracy of effusion cytology. 
Part II of this study presents the ability of the same immunocytochemical markers and DNA-cytometry to detect malignant epithelial and mesothelial cells in diagnostically equivocal effusions.

\section{Materials and methods}

\subsection{Specimens and patient population}

Subject of our study were 154 effusions of the serous cavities with cytologically tumour cell positive (101) and negative (53) diagnoses. 107 pleural, 43 peritoneal, three pericardial effusions and one coul de sac aspiration were routinely investigated between April 1996 and December 1997 in the Institute of Cytopathology. The patients were from the University Hospital of Düsseldorf as well as from hospitals of the surrounding area. Effusions were selected as containing a sufficient number of reactive or malignant mesothelial or epithelial cells for application of adjuvant methods.

\subsection{Staining of specimens}

Native materials were centrifuged at $300 \mathrm{~g}$ for $5 \mathrm{~min}$. Four slides were processed by pipetting a drop of the sediment, containing some residual liquid on each slide. A second slide each was used to top the first four in order to produce eight smears. Three of these slides were air-dried and stained according to MayGrünwald-Giemsa, one of these was used for DNAimage cytometry. The other slides were prepared for Papanicolaou staining and subsequent immunocytochemistry (ICC) by immediate fixation in Delaunay's fixative [21] $(500 \mathrm{ml}$ ethanol plus $500 \mathrm{ml}$ aceton (Riedel-de-Haën, Seelze, Germany, no. 24201) plus ten drops of $1 \mathrm{M}$ trichloroacetic acid (Merck, Darmstadt, Germany, no. 1.00807.0100)) for $10 \mathrm{~min}$. Coverslips were removed in xylene before immunocytochemistry or DNA-cytometry were performed.

\subsection{Cytological diagnosis}

Specimens were evaluated according to generally accepted diagnostic criteria $[7,11,15,42]$ by an experienced cytotechnician (K. K.) and by cytopathologists (H. M. or A. B.) as well. The following categories of cytologic diagnoses were chosen: "insufficient" for specimens without any or with exclusively autolytic or necrotic cells, "negative" for inconspicuous, reactive or inflammatory cellular changes, "doubtful" in cases with atypical cellular activation or degeneration, "suspicious" if only sparse abnormal cells were seen or the diagnostic criteria for malignancy were only vague and "positive" for effusions containing unequivocal malignant cells $[7,16]$.

\subsection{Immunocytochemistry}

The application of immunocytochemistry on cytological specimens was developed by P. D. and B. B. in the Division of Cytopathology, Institute of Pathology, University of Basel [21]. For immunocytochemistry on slides previously fixed according to Delaunay and stained according to Papanicolaou cells of interest were marked by felt-tip pen. Coverslips were then removed in xylene. If only few suspicious cells were present the slides were divided into two or three sections using a DakoPen (Dako, Glostrup, Denmark, no. S2002) so that several antibodies could be applied simultaneously on the same slide. The Avidin-BiotinComplex method (ABC) [21] was applied for immunological reaction. The incubations were carried out in a horizontal position of the slides in a humid chamber. All other steps were carried out in an upright position of slides in cuvettes. Endogenous peroxidase activity was stopped by incubation with $1 \mathrm{ml} \mathrm{H}_{2} \mathrm{O}_{2}$ (30\% Perhydrol, Merck, Darmstadt, Germany, no. 1.07209.0250) in $100 \mathrm{ml}$ methanol (Merck, Darmstadt, Germany, no. 1.06008.250) for $30 \mathrm{~min}$ at room temperature (RT). After shortly rinsing the slides manually three times in phosphate buffered saline (PBS, Sigma, Deisenhofen, Germany, no. D-1408) they were then placed twice in PBS at RT for 10 min each. They were then incubated in normal (horse) serum (for mouse antibodies) (225 $\mu \mathrm{l}: 15 \mathrm{ml}$ aqua dest.) (Vector, Camon Laboratory Service, Wiesbaden, Germany, no. S-2000) for $20 \mathrm{~min}$ at RT. Letting the residual liquid drip off the slide by slanting, the slides were incubated with the primary antibody (antibodies applied, as well as their specifications and dilutions are described below) for $12 \mathrm{~h}$ at $25^{\circ} \mathrm{C}$. The slides were then rinsed twice in $0.5 \mathrm{M}$. TRIS (tris(hydroxymethyl)aminomethan), $\mathrm{pH}=7.6$ in PBS 1 : 10 for $5 \mathrm{~min}$ at RT each. This was followed by incubation with the link (biotinylated) antibody (LINK) [21] (Vector, Camon Laboratory Service, Wiesbaden, Germany, no. BA-2000) (30 min at RT) and once again the slides were rinsed twice in $0.5 \mathrm{M}$. TRIS in PBS $1: 10$ for 5 min at RT. The slides were then incubated with the ABC-Elite-Standard [21] (Vectastain, Camon Laboratory Service, Wiesbaden, Germany, no. PK-6100) for further $30 \mathrm{~min}$ at RT and 
once again rinsed twice in TRIS $0.5 \mathrm{M}$ (Boehringer, Mannheim, Germany, no. 812838) in PBS 1 : 10 for $5 \mathrm{~min}$ at RT. The substrate-chromagen-reagent AEC (3-amino-9-ethylcarbazole) [21] (Sigma, Deisenhofen, Germany, no. A5754) was then applied for $40 \mathrm{~min}$ at RT followed by rinsing twice in sterile aqua dest. for $5 \mathrm{~min}$ at RT each. Counterstaining was performed with Mayers Haematoxylin (1 min RT) rinsed under tap water and coverslipped in Aquatex (water-based mounting medium, Merck, Darmstadt, Germany, no. 1.08562).

Absence of staining in other than the cell type under consideration served as negative controls. (As we realize this was not an optimal approach, we meanwhile apply isotypic Ig as negative controls.) Positive staining of other cases with the same antibody simultaneously applied served as positive control.

Specifications and applied dilutions (in PBS) of primary antibodies towards:

Ber-EP4 1:200, Dako, Glostrup, Denmark, no. M804; marks surface and cytoplasmic glycoprotein,

B72.3 1:1600, BioGenex Lab, S. Remon, C., USA, no. MAU054UC; marks cytoplasmic tumour-associated glycoprotein,

CA125 1:80, Cis bio international, Sur Yvette, France, no. CA125II; marks membrane hihmolecular mucin,

CEA $\quad 1: 200$, Dako, Glostrup, Denmark, no. M707; marks cytoplasmic carcinoembryonic antigen, a heavily glycosilated protein,

Desmin $\quad 1: 500$, Dako, Glostrup, Denmark, no. M0760; marks cytoplasmic intermediate filaments,

EMA $1: 1600$, Dako, Glostrup, Denmark, no. M0613; marks epithelial membrane antigen, a human milk fat globulin,

HBME-1 1:1400, Dako, Glostrup, Denmark, no. M3505; marks cytoplasmic intermediate filaments,

Leu-M1 1:1000, Dako, Glostrup, Denmark, no. M0733; marks cytoplasmic oligosaccharid,

Lu5 1:2500, Biochemicals AG, Augst, Switzerland, no. T-1302; marks cytoplasmic cytokeratin,
MAC 387 1:2000, Dako, Glostrup, Denmark, no. M0747; marks cytoplasmic myeloid-histiocyte,

MNF 116 1:500, Dako, Glostrup, Denmark, no. M0821; marks cytoplasmic cytokeratin,

Vimentin $1: 600$, Dako, Glostrup, Denmark, no M725; marks cytoplasmic intermediate filaments.

\subsection{Evaluation of immunocytochemically stained slides}

Slides were evaluated independently by two experts (M. K. and H. M.). The following semiquantitative score was applied: "-" (negative) for no cellular staining detectable. " $(+)$ " (negative) for less than $5 \%$ of the respective cell population weakly stained. "+" (positive) for 6-30\% of the respective cell population stained, "++" (positive) for $31-70 \%$ of the respective cell population stained and " +++ " (positive) for 71$100 \%$ of the respective cell population stained. Staining intensity was usually strong but played no role in scoring the quantity of immunocytochemical reaction. To exclude false positive results due to artificial or unspecific staining, less than $5 \%$ of weakly stained cells (" $(+)$ ") was declared as the cut-off value for interpretation of staining results as negative. Antibody reactions were evaluated per case, without knowledge of patient follow-up in order to avoid any bias in decision making. Results were then discussed between both observers. There were no discrepancies in the interpretation of staining results as positive or negative.

Prevalence of staining was thus evaluated for each antibody in reactive mesothelial cells, macrophages and in the respective abnormal cell population in each effusion. Possible unspecific cellular staining of lymphocytes and granulocytes was registered.

\subsection{Staining of specimens for DNA-cytometry}

Slides prestained according to May-GrünwaldGiemsa were uncovered in xylene and Feulgen stained in a temperature-controlled staining machine with Schiff's reagent according to the protocol described earlier $[13,14,56]$. In brief, after rehydration in decreasing ethanol concentrations and re-fixation in buffered $10 \%$ formalin, $5 \mathrm{~N} \mathrm{HCl}$ for acid hydrolysis was applied at $27^{\circ} \mathrm{C}$ for one hour, followed by staining in Schiff's reagent (Merck, Darmstadt, Germany, no. 1.09033.0500) for another hour, followed by rinsing in 
$\mathrm{SO}_{2}$-water and dehydration at increasing ethanol concentrations. The slides were then covered with Entellan (Merck, Darmstadt, Germany, no. 1.07961.0500).

\subsection{Measurement}

Measurements of nuclear DNA were performed as described in our previous papers $[13,14,38,56]$. In brief, 30 lymphocytes were measured as reference cells, meeting a coefficient of variation of $\leqslant 5 \%$. Subsequently, if present, 300 reactive mesothelial, atypical or abnormal cells were measured per specimen interactively at random. The ZEISS CYRES workstation (Zeiss, Jena, Germany) was used for measurements. It consists of a conventional light microscope Axioplan 2 (Zeiss, Jena, Germany) with a $40 \times$ objective; NA 0.75; Köhler illumination was performed to reduce the stray light and a $570 \mathrm{~nm}$ interference filter $\pm 10 \mathrm{~nm}$ half value width was used. A CCD black and white camera (VarioCam (Modell CCIR), PCO Computer Optics, Kehlheim, Germany) with 572 lines resolution was adapted to the microscope. The images were analyzed using a PC Pentium I processor and framegrabber (Kontron, Eching, Germany) and VGA screen, mouse and laser printer. The performance of the system meets the standards of the European Society for Analytical Cellular Pathology (ESACP) task force on standardization of diagnostic image cytometry [14, 38]. Segmentation was performed automatically on individual nuclei by grey level thresholding taking the local background into consideration. The glare error was corrected by software as previously described [13], at a rate of $2.2 \%$.

The data were diagnostically interpreted as described in a previous paper [56]. DNA-aneuploidy was assumed, if the DNA-index of an abnormal stemline was $<0.925>1.075$ or $<1.80>2.20$ or $<3.60>$ 4.40 , a coefficient of variation (CV) of the first stemline was $\geqslant 10 \%$ and/or cells $>9 \mathrm{c}$ occurred (9c exceeding events ( $9 \mathrm{c} \mathrm{EE})$ ).

\subsection{Validation of cytologic diagnoses}

According to patient follow-up (periods of 6-18 months) the investigated effusions were classified as either containing malignant cells or not. Patient histories were only accepted for evaluation if retrospectively presenting sufficient evidence for the presence or absence of tumour cells in effusions. These revealed either histologic follow-up of a tumour of the serous membranes itself $(43 / 154=27.9 \%)$ or of the respective primary tumour (with clinical evidence of a malignant tumour) $(57 / 154=37.0 \%)$ or clinical evidence for the malignant or benign nature of the effusion, applying advanced diagnostic techniques (i.e., radiology, computed tomography, laparoscopy, $54 / 154=35.1 \%$ ) was found. Patients presenting tumour cell positive effusions revealed the following primary tumours: carcinomas of the breast (17), the ovary (22), the endometrium (1), the lip (1), the thyroid (1), the lung (19), the esophagus (1), the stomach (2), the colon sigmoideum (2), the pancreas (1), the kidney (1), the urothelium (1), as well as carcinomas of unknown primary (CUP) (18). Furthermore, malignant mesotheliomas (without further histologic classification) (14) as well as a non-Hodgkin-lymphoma (1) occurred. Although cytologically mimicking cells of a carcinomatosis, the effusion due to lymphoma was excluded from the evaluation as immunocytochemical markers were selected to solely distinguish between epithelial and mesothelial cells and not cells of lymphoma. According to histologic follow-up, among the tumour cell positive effusions, there were two cytodiagnostically seemingly "false positive" cases both due to chronic unspecific pleuritis. Both cases undoubtedly revealed tumour cells even on second-look inspection. This was confirmed by immunocytochemistry and DNA-cytometry. Patients presenting tumour cell negative effusions showed the following basic diseases: pneumonia/pleuritis (17), congestive heart failure (21), renal insufficiency (1), cirrhosis of the liver (6), endometriosis (2), ovarian cyst (1), gastritis (1), pancreatitis (1), insufficiency of rectum anastomosis (1) and cholecystitis (1). Furthermore, there was one cytologically "false negative" pleural effusion due to a carcinoma of the lung, which was detected as tumour cell positive by immunocytochemistry and by DNAcytometry. It was re-evaluated as tumour cell positive on cytologic second-look inspection.

A tumour cell positive effusion was considered as truly positive if it was confirmed by medical report with one of the above mentioned techniques. In two cases with positive cytological diagnosis but without further clinical, radiological or histopathological evidence of a malignant tumour re-evaluation of the slides (by two expert cytopathologists: H. M. and A. B.) led to a definitively positive morphological diagnosis, confirmed by immunocytochemistry and DNA-cytometry.

To evaluate the specificity of the method, the reports on patients with any non-positive diagnosis in effusion cytology were checked for further evidence of a malignant tumour. If there were no discrepancies between 
the cytologic diagnosis and the medical report, the diagnosis was considered as truly negative. A cytologic diagnosis was also considered as truly negative if clinical evidence of a non-tumourous origin of the effusion was given, also despite the fact that a patient might have had a malignant tumour as well. It was classified as false negative $(n=1)$ if a malignant tumour was diagnosed clinically, radiologically or histopathologically during the patients follow-up period and if no non-tumourous origin of the effusion was found. By re-evaluation of slides tumour cells were found on second-look inspection.

After comparing results of cytology, immunocytochemistry and DNA-cytometry with histologic followup diagnoses seven cases were re-evaluated. In these cytologically correctly positive diagnosed cases histologic follow-up showed discrepancies with combined cytological-, immunocytochemical- and DNAcytometric diagnoses (these three methods were in accordance) concerning the histogenetic tumour typing. In these cases histologic specimens were re-evaluated and the primary diagnosis doubted. Five patients with the histologic diagnosis "suspicious for mesothelioma" were re-evaluated as carcinomatoses, as cytology, as well as both adjuvant methods clearly demonstrated the epithelial origin of the cells and histology was also consistent with this finding. One histologic diagnosis of carcinomatosis was re-evaluated as mesothelioma, as cytology, as well as both adjuvant methods clearly demonstrated the mesothelial origin of the cells and histology was consistent with this finding as well. Specimens of one further patient with history of a chronic lymphatic leukemia were re-evaluated and diagnosed to contain cells of an adenocarcinoma as all three methods: cytology, immunocytochemistry and DNA-cytometry were in accordance, so that it must be concluded that the patient has an occult adenocarcinoma additionally to his leukemia.

\section{Results}

Prevalences of cellular staining for different antibodies in benign and malignant effusions (totally as well as separately for carcinomatoses and mesotheliomas) are given in Figs 1-4.

Ber-EP4, anti-Leu-M1, -CEA and -EMA were the immunocytochemical markers with the most distinct results in the detection of malignant cells and the differential diagnosis between benign and malignant mesothelial cells and cells of metastatic carcinomas (Table 1). While staining for Ber-EP4 in tumour cells had a prevalence of $82.2 \%$ in malignant effusions, no cell type reacted with this marker in benign effusions $(0 \%)$. Within malignant effusions prevalence was $95.4 \%$ in metastatic carcinomas and $0 \%$ in mesotheliomas. Ber-EP4 stained adenocarcinomas, as well as squamous cell- and small cell carcinomas. The four Ber-EP4 negative malignant effusions were due to ovarian $(n=2)$, thyroid $(n=1)$ and urothelial ( $n=1)$ cancers, this number of cases being too small to detect any correlation between different types of pri-

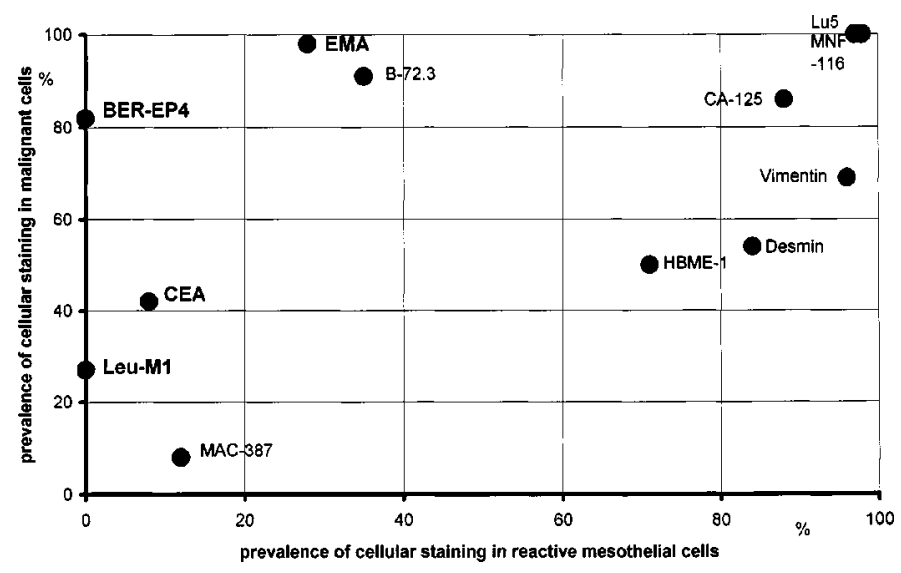

Fig. 1. Prevalence of cellular staining by twelve different immunocytochemical markers in malignant cells in 101 tumour cell positive effusions as opposed to reactive mesothelial cells in 53 tumour cell negative effusions.

Note: Ber-EP4 marks a surface and cytoplasmic glycoprotein, Lu5 and MNF 116 mark cytoplasmic cytokeratins, EMA (epithelial membrane antigen) marks a human milk fat globulin, B72.3 a cytoplasmic tumour-associated glycoprotein, Leu-M1 a cytoplasmic oligosaccharid, CEA (carcinoembryonic antigen) a heavily glycosilated cytoplasmic protein, CA-125 a membrane highmolecular mucin, HBME-1 cytoplasmic mesenchym, vimentin and desmin mark cytoplasmic intermediate filaments, and MAC 387 marks cytoplasmic myeloid-histiocyte structures. 


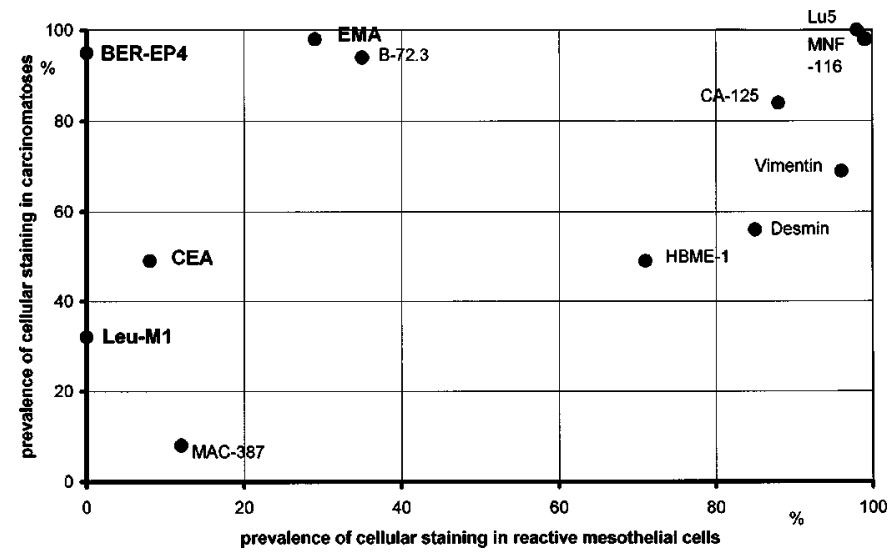

Fig. 2. Prevalence of cellular staining by twelve different immunocytochemical markers in carcinomatoses in 87 tumour cell positive effusions as opposed to reactive mesothelial cells in 53 tumour cell negative effusions.

Note: (see Fig. 1).

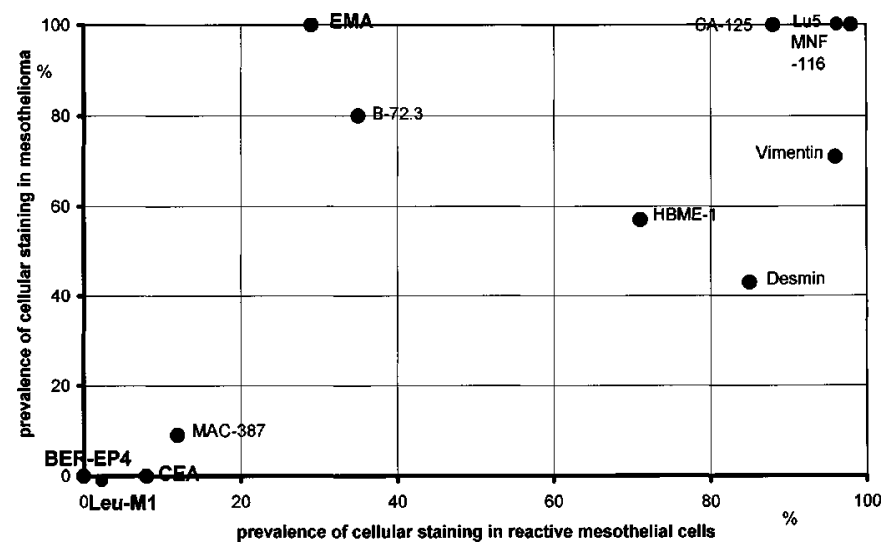

Fig. 3. Prevalence of cellular staining by twelve different immunocytochemical markers in mesotheliomas in 14 tumour cell positive effusions as opposed to reactive mesothelial cells in 53 tumour cell negative.

Note: (see Fig. 1).

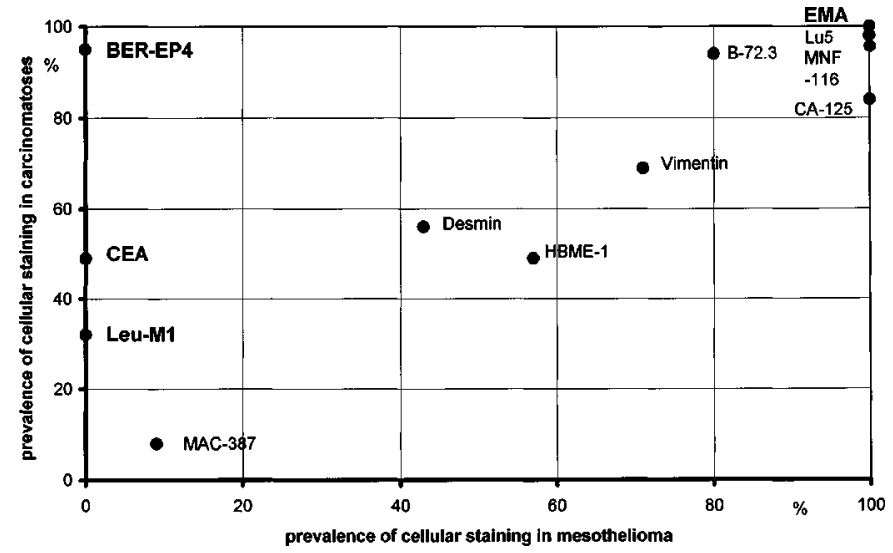

Fig. 4. Prevalence of cellular staining by twelve different immunocytochemical markers in carcinomatoses in 87 tumour cell positive effusions as opposed to in mesotheliomas in 14 tumour cell positive effusions.

Note: (see Fig. 1). 
Table 1

Prevalence of cellular staining by four antibodies in effusions

\begin{tabular}{lcccc}
\hline Follow-up diagnoses & Ber-EP4 & Leu-M1 & EMA & CEA \\
\hline $\begin{array}{l}\text { Without tumour cells } \\
\quad n=53\end{array}$ & $0 \%(0 / 53)$ & $0 \%(0 / 53)$ & $28.8 \%(15 / 52)$ & $7.7 \%(4 / 52)$ \\
$\begin{array}{l}\text { Carcinomatosis } \\
\quad n=87\end{array}$ & $95.4 \%(83 / 87)$ & $32.2 \%(28 / 87)$ & $97.7 \%(85 / 87)$ & $49.4 \%(42 / 85)$ \\
Mesothelioma & & & \\
$\quad n=14$ & $0 \%(0 / 14)$ & $0 \%(0 / 14)$ & $100 \%(14 / 14)$ & $0 \%(0 / 14)$ \\
$\begin{array}{l}\text { Total with tumour cells } \\
n=101\end{array}$ & $82.2 \%(83 / 101)$ & $27.7 \%(28 / 101)$ & $98.0 \%(99 / 101)$ & $42.4 \%(42 / 99)$ \\
\hline
\end{tabular}

mary tumours and absent Ber-EP4 staining. Anti-LeuM1, -EMA and -CEA revealed prevalences of 27.2, 98.0 and $42.6 \%$ in malignant cells in effusions (32.2, 97.7 and $49.4 \%$ in metastatic carcinomas and 0,100 and $0 \%$ in mesotheliomas). Prevalences of staining of reactive mesothelial cells in benign effusions was $0 \%$ for anti-Leu-M1, $28.8 \%$ for -EMA and, $7.6 \%$ for -CEA, respectively. There was no special pattern of staining of antibodies to be detected that could be considered as distinctive for a certain cell type. Especially EMA did not consistently show a thick membrane pattern in mesotheliomas (as sometimes mentioned in the literature [7]), but instead sometimes also showed it in reactive mesothelial cells or carcinomas. Ber-EP4 as well seldomly stained in a thick membrane pattern without specificity for a certain cell type. Of the four above mentioned antibodies anti-Leu-M1 was the only marker unspecifically staining granulocytes. No staining of lymphocytes or macrophages was seen.

In effusions containing malignant cells immunocytochemistry was able to correctly differentiate between metastatic carcinomas and mesotheliomas in 98.0\% (99/101) by applying Ber-EP4 and/or anti-LeuM1 which were both exclusively present in cells of metastatic carcinomatoses and/or -CEA which was in malignant effusions only present in metastatic carcinomatoses.

Table 1 demonstrates in detail the results of immunocytochemistry in the differential diagnosis of benign and malignant mesothelial and metastatic carcinoma cells. While anti-Ber-EP4 showed positive cellular staining in $95.4 \%$ of malignant effusions due to metastatic carcinomatoses, it never showed positive staining of any cell type in benign effusions $(0 \%)$ or mesotheliomas $(0 \%)$. Positive cellular staining $(+,++,+++)$ by anti-Leu-M1 was exclusively seen in effusions due to metastatic carcinomatoses (in $32.2 \%$ ). Anti-EMA showed positive cellular staining in all three differential diagnoses mentioned above, but nearly $100 \%$ positivity of all abnormal cells is only seen in malignant effusions. Cellular staining for antiCEA was observed in benign effusions $(7.6 \%)$ and those due to carcinomatoses $(49.9 \%)$, but not in effusions due to mesotheliomas $(0 \%)$.

The other immunocytochemical markers were not as helpful distinguishing benign or malignant mesothelial cells or those from carcinomatoses. In spite of optimized protocols they either showed constant positive (anti-Lu5 and anti-MNF 116) or negative (antiMAC 387) staining of both reactive mesothelial cells in benign as well as abnormal cells in malignant effusions due to metastatic carcinoma as well as to mesothelioma and therefore did not contribute to the differential diagnosis. Or they revealed strong differences in scoring (from negative to " +++ " positive) (anti-CA125, anti-desmin and anti-vimentin). Furthermore, technical difficulties were encountered such as improper fixation or overstaining and therefore evaluation was almost impossible (anti-B72.3, anti-HBME-1) leading to high interobserver variability. Some of these antibodies showed staining of other cell types in effusions, making interpretation difficult: anti-Leu-M1 (strongly), MAC387 (strongly) and -CEA (weakly) stained neutrophil granulocytes and monocytes; MAC387 of course stained macrophages as well.

Combinations of staining results of the four most helpful immunocytochemical markers: Ber-EP4, antiLeu-M1, -EMA and -CEA were considered (Table 1). Their ability to detect malignant cells in effusions, without loss of specificity of $100 \%$, was regarded. BerEP4 as the most sensitive and specific marker was able to detect abnormal cells in $82.2 \%(83 / 101)$ of the malignant effusions. Of the 18 cases not staining positive for Ber-EP4, 14 were effusions due to mesothelioma, four to carcinomatoses. Anti-Leu-M1 as a marker for the detection of malignant cells found only one additional carcinoma. 
Table 2

Prevalence of DNA-aneuploidy in tumour cell positive and negative effusions

\begin{tabular}{lcccc}
\hline DNA-ploidy-status & \multicolumn{4}{c}{ Follow-up diagnosis } \\
\cline { 2 - 5 } & Without tumour cells & Carcinomatoses & Mesothelioma & Total with tumour cells \\
& $n=52(100 \%)$ & $n=87(100 \%)$ & $n=14(100 \%)$ & $n=101$ \\
\hline Non-aneuploid & $52(100.0 \%)$ & $4(4.6 \%)$ & $6(42.9 \%)$ & $10(9.9 \%)$ \\
Aneuploid & $0(0.0 \%)$ & $83(95.4 \%)$ & $8(57.1 \%)$ & $91(90.1 \%)$ \\
\hline
\end{tabular}

Table 3

Prevalence of cellular staining by Ber-EP4 and DNA-aneuploidy in effusions

\begin{tabular}{lccc}
\hline Follow-up diagnoses & ICC: BER-EP4 & DNA-ICM: aneuploidy & ICC and/or DNA-ICM \\
\hline $\begin{array}{l}\text { Without tumour cells } \\
n=53\end{array}$ & $0 \%(0 / 53)$ & $0 \%(0 / 53)$ & $0 \%(0 / 53)$ \\
$\begin{array}{l}\text { Carcinomatosis } \\
n=87\end{array}$ & $95.4 \%(83 / 87)$ & $95.4 \%(83 / 87)$ & $100 \%(87 / 87)$ \\
$\begin{array}{l}\text { Mesothelioma } \\
n=14\end{array}$ & $0 \%(0 / 14)$ & $57.1 \%(8 / 14)$ & $57.1 \%(8 / 14)$ \\
$\begin{array}{l}\text { Total with tumour cells } \\
n=101\end{array}$ & $82.2 \%(83 / 101)$ & $90.1 \%(91 / 101)$ & $94.1 \%(95 / 101)$ \\
\hline
\end{tabular}

Regarding Figs 1-4 it can be concluded that it is possible to choose panel combinations resulting in an increase of sensitivity yet associated with a loss of specificity.

None of the DNA-histograms of mesothelial cells in non-malignant, inflammatory or reactive effusions revealed any of the above mentioned criteria of DNAaneuploidy and were therefore all interpreted as DNAeuploid (Table 2). This corresponds to a prevalence of $100 \%$ of the marker DNA-euploidy in reactive mesothelial cells.

The DNA-histograms of effusions containing malignant cells presented one, two or three aspects of DNAaneuploidy mentioned above. On this basis $83 / 87$ of the effusions due to metastatic carcinomas and $8 / 14$ due to mesotheliomas were DNA-aneuploid (Table 2). This corresponds to a prevalence of DNA-aneuploidy in malignant cells in effusions of $95.4 \%$ for metastatic carcinomatoses and $57.1 \%$ for mesotheliomas. The detection rate of DNA-aneuploidy depends on the application of one to three different algorithms. Nuclear DNA-values $>9 \mathrm{c}$ represented the most frequent aspect of DNA-aneuploidy (86.1\%). Additionally accepting an abnormal stemline, present in $79.2 \%$, as an aspect of DNA-aneuploidy, prevalence increased to $90.1 \%$. Applying a $\mathrm{CV} \geqslant 10 \%$ as another aspect of DNAaneuploidy, present in $21.8 \%$ of cases, did not result in a further increase of detection rate.

While $64.3 \%$ (9/14) of mesotheliomas showed their greatest DNA-stemline within the range of $1.80 \mathrm{c}$ and
2.20 c, only $9.2 \%(8 / 87)$ of carcinomatoses showed their greatest stemline in this region.

Combining immunocytochemistry solely applying Ber-EP4 as marker with DNA-cytometry leads to an increase in detection of cells of carcinomatoses from $95.4 \%$ only applying the former and $95.4 \%$ the latter to $100 \%$ applying both methods (Table 3 ). For malignant mesothelioma cells the detection rate in effusions is $0 \%$ solely applying immunocytochemistry and $57.1 \%$ DNA-cytometry (Table 3).

\section{Discussion}

We have investigated 101 tumour cell positive and 53 -negative effusions concerning immunocytochemical staining for 12 antibodies as well as the marker DNA-aneuploidy by DNA-image cytometry.

Staining for Ber-EP4 had a prevalence in tumour cells of $82.2 \%(95.4 \%$ in metastatic carcinomas and $0 \%$ in mesotheliomas). Since our analysis achieved a specificity of $100 \%$ of negative staining for the immunocytochemical marker Ber-EP4 in benign effusions, false positive diagnosis due to unspecific cellular staining in reactive effusions are not to be expected. We propose that staining of cells for Ber-EP4 in effusions may be used as a highly specific and sufficiently sensitive marker for neoplastic epithelial cells. This marker can furthermore differentiate between cells of metastatic carcinoma and mesothelioma in $98.0 \%$. 
The application of immunocytochemistry as a panel obviously reflects insufficient sensitivity and specificity of single markers which were so far applied. Our study on prevalences of 12 different antibodies in cells of benign and malignant effusions demonstrates that the sole use of Ber-EP4 results in an excellent detection rate of malignant epithelial cells. We therefore do not recommend the use of a panel of antibodies for the detection of malignant cells in effusions. For the differential diagnosis between malignant cells due to carcinomatoses and mesotheliomas we suggest the additional use of anti-Leu-M1 and/or -CEA which were only positive in cells of metastatic carcinomatosis. Recently, we additionally apply anti-calretinin to differentiate between mesothelial and epithelial cells in effusions. It seems that anti-calretinin is a useful additional marker for the differentiation between carcinomatoses and mesotheliomas as it is a highly sensitive and rather specific marker for reactive and malignant mesothelial cells. The reduction of an immunocytochemical panel to 1-2 antibodies helps to reduce laboratory and personnel costs without loss of sensitivity and specificity. We do not recommend the application of an immunocytochemical panel using different coefficients attributed to various antibodiesfor the differentiation between metastatic carcinomatosis and mesothelioma $[24,25]$.

Altogether, immunocytochemistry as an adjuvant method seems to be a valuable tool for the detection of malignant cells in effusions and in the differential diagnosis between metastatic carcinoma and mesothelioma. Thus, this method should be able to increase diagnostic accuracy of conventional effusion cytology.

Reasons for differences in prevalences of, e.g., BerEP4 positive staining (32-100\%) in the literature as well as compared to our study, are the following: while we applied Delaunay fixation on smears of effusion sediments followed by staining according to the Avidin-Biotin-Complex method other authors have used many different types of fixations on various types of cell preparations also applying different staining methods. All of the factors influence immunocytochemical staining results. We suggest to apply immunocytochemical methods only according to standardized protocols optimized especially for effusion specimens. Merely applying a protocol established only for histologic material without optimal fixation and tested antibody concentrations for cytologic specimens will lead to unsatisfactory results. Different study designs (i.e., patient populations, selection of effusions for cytologic evaluation) may also lead to different results.
A further important aspect concerning the interpretation of immunocytochemical staining in effusions is the "cut-off" level for acceptance of cellular staining as positive. Even if an antibody is absolutely specific in detecting a certain cell type, there remains a risk of unspecific staining of a few cells, e.g., due to cellular degeneration or necrosis or to serum precipitates. Interpretation of staining results may also be due to equivocal morphological typing of cells. Therefore, a "cutoff" level of $>5 \%$ staining of the respective population of cells is recommended to accept a staining result as positive, otherwise false positive results may occur.

Qualifications for the exact judgement of the efficacy of an antibody to detect malignant cells in effusions are: cytology-adapted techniques of immunocytochemistry [21], the interpretation of staining results by a semiquantitative scoring scheme in different cell types and the calculation of prevalences of staining per cell type and antibody. These are the prerequisites to then compare the efficacy of antibodies in a panel. With these data we now may conclude that a panel of immunocytochemical markers is not necessary.

Furthermore, in our study prevalence of immunocytochemical markers is compared with that of DNAaneuploidy in tumour cell positive and negative effusions.

The diagnostic value of DNA-cytometry for the identification of malignant cells in effusions has been demonstrated by our group in two previous studies [56, 57]. We proposed the combined use of three different algorithms for the identification of DNA-aneuploidy: position of any DNA-stemline, $\mathrm{CV}$ of the first stemline $\geqslant 10 \%$ and occurrence of cells $>9 \mathrm{c}$.

Considering the diagnostic interpretation of DNAcytometric results, we recommend strict consideration of the standards of the European Society for Analytical Cellular Pathology (ESACP) for diagnostic DNAimage cytometry $[14,38]$, otherwise, false positive diagnoses may occur.

In this study our measurements identified DNAaneuploidy in $90.1 \%$ of specimens from malignant effusions $(95.4 \%$ in metastatic carcinomas and $57.1 \%$ in mesotheliomas). Since our analysis achieved a specificity of $100 \%$ for the marker DNA-euploidy to confirm absence of malignant cells in effusions, false positive diagnoses in reactive effusions are not to be expected. We therefore believe that DNA-image cytometry is also able to increase diagnostic accuracy of effusion cytology. In effusions containing malignant cells the location of the greatest stemline by DNAcytometry is able to contribute to the differential di- 
agnosis between metastatic carcinomas and mesotheliomas [56]. While $64.3 \%$ of mesotheliomas showed their greatest stemline within the range of $1.80 \mathrm{c}$ and $2.20 \mathrm{c}$, only $9.2 \%$ of carcinomatosis did. In our previous study [56] none of the carcinomatoses showed their greatest stemline at this location.

Apart from our study three other authors have suggested the combined use of immunocytochemical markers and DNA-cytometry. While Croonen et al. [19], and Joseph et al. [41], suggest the use of DNAflow cytometry as opposed to DNA-image cytometry, which is principally a good method for the detection of aneuploid DNA-stemlines, it should be considered that rare events ( $9 \mathrm{c}$ exceeding events) are usually not detected by DNA-flow cytometry. Matter-Walstra and Kraft [51] introduced the combined use of staining for Ber-EP4 and DNA-image cytometry. Principally, this is a good methodological procedure, but the authors used $5 \mathrm{c}$ ER $\geqslant 10 \%$ as the only criterion for DNA-aneuploidy. Yet, this is not an acceptable marker in cells undergoing euploid polyploidization, like mesothelial cells, as they normally reveal cells $>5 \mathrm{c}$ EE [12,57]. Therefore, cells with DNA-content between $4 c$ and $8 c$ must regularly be expected.

Problems arise concerning the interpretation of the follow-up of patients with cytologically tumour cell positive and -negative effusions. At present there is no general agreement as to the "golden standard" for evaluation of the diagnostic accuracy of immunocytochemical or DNA-cytometric diagnoses. This aspect is handled controversially in the literature. Some studies report on histological, others only on clinical evidence of the basic disease. Most authors merely compare immunocytochemical or DNA-cytometric results with routine cytologic diagnoses. In our study the immunocytochemical finding of malignant epithelial cells or the cytometric finding of DNA-aneuploidy was classified as correctly positive if patient follow-up revealed histologic or clinical evidence for a malignant nature of the effusion. They were classified as correctly negative if the patient follow-up revealed histologic or clinical evidence for a benign nature of the effusion. We felt it necessary to re-evaluate seven cases concerning their histogenetic classification of malignant cells. As adjuvant methods indicated the origin of the specimens as epithelial or mesothelial, histology had to be re-evaluated.

Part II of this study demonstrates the application of these adjuvant methods in diagnostically equivocal effusions.

\section{Conclusion}

We assume that with cytology-adapted techniques of immunocytochemistry, standardized interpretation of staining results as well as with improved precision of DNA-cytometry and application of different algorithms for histogram analysis both methods may help to unequivocally detect a high percentage of tumour cells in cytologically equivocal effusions. A combination of both methods will hopefully result in an increased sensitivity for the detection of malignant cells and thus decrease the rate of cytologically definitely doubtful effusions. In tumour cell positive effusions they may furthermore help to differentiate between metastatic carcinomatoses and mesotheliomas.

\section{Acknowledgement}

We wish to thank all clinicians who generously supported us by providing patient follow-up data. Furthermore, we would like to express our thanks to Mrs B. Buckstegge and Mrs H. Müschenborn for excellent preparation of slides and to Mrs D. Shittu for expert typing of the manuscript.

\section{References}

[1] I.V. Abramenko, D.F. Gluzman, L.M. Sklyarenko and G.V. Pisnyachevskaya, Immunocytochemical staining of cells in 153 pleural effusions with a panel of monoclonal antibodies and lectins, Anticancer Res. 11 (1991), 629-634.

[2] A. Al-Nafussi and P.J. Carder, Monoclonal antibodies in the cytodiagnosis of serous effusions, Cytopathology 1 (1990), 119128.

[3] V. Ascoli, S. Taccogna, C. Carnovale-Scalzo and F. Nardi, Utility of cytokeratin 20 in identifying the origin of metastatic carcinomas in effusions, Diagn. Cytopath. 12 (1995), 303-308.

[4] V. Ascoli, C. Carnovale-Scalzo, S. Taccogna and F. Nardi, Utility of HBME-1 immunostaining in serous effusions, $C y$ topathology 8 (1997), 328-335.

[5] P. Athanassiadou, P. Athanassiades, D. Lazaris, K. Kyrkou, E. Petrakakou and D. Aravantinos, Immunocytochemical differentiation of reactive mesothelial cells and adenocarcinoma cells in serous effusions with the use of carcinoembryonic antigen and fibronectin, Acta Cytol. 38(5) (1994), 718-722.

[6] M.C.P. Barberis, M. Faleri, F. Veronese, C. Casadio and G. Viale, Calretinin-A selective marker of normal and neoplastic mesothelial cells in serous effusions, Acta Cytol. 41 (1997), 1757-1761.

[7] C.W.M. Bedrossian, Malignant Effusions. A Multimodal Approach to Cytologic Diagnosis, Igaku-Shoin Medical Publishers, New York, Tokyo, 1994 
[8] C.W.M. Bedrossian, Diagnostic problems in serous effusions, Diagn. Cytopathol. 19(2) (1998), 131-137.

[9] J.E. Beesley, Immunocytochemistry: A Practical Approach, Oxford University Press, Oxford, New York, Tokyo, 1993, pp. $15-42$.

[10] M. Beuzelin-Yvraut, A. Bourguignat, E. Phillips, A. Roseto and E. Osinaga, Immunocytological analysis of the Tn associated antigen $83 \mathrm{D}_{4}$ in serous effusions from patients with cancer: Comparison with Tn soluble glycoprotein, J. Clin. Pathol. 48 (1995), 433-437.

[11] M. Bibbo, Comprehensive Cytopathology, W.B. Saunders Company, Philadelphia, 1991, pp. 541-614.

[12] S. Biesterfeld, K. Gerres, G. Fischer-Wein and A. Böcking, Polyploidy in non-neoplastic tissues, J. Clin. Pathol. 47 (1994), $38-42$.

[13] A. Böcking, DNA measurements: when and why?, in: Compendium on Quality Assurance, Proficiency Testing and Workload Limitations in Clinical Cytology, G.L. Wied, C.M. Keebler, D.L. Rosenthal, U. Schenk, T.M. Somrak and G.P. Vooijs, eds, Tutorials of Cytology, Chicago, 1995, pp. 170-188.

[14] A. Böcking, F. Giroud and A. Reith, Consensus report of the European Society for Analytical Cellular Pathology task force on standardization of diagnostic DNA-image cytometry, Analyt. Cell. Pathol. 8 (1995), 67-74.

[15] A. Böcking, Cytopathologie, in: Lehrbuch der Histopathologie, 12th edn, C. Thomas, ed., F.K. Schattauer Verlag, Stutgart, New York, 1998, pp. 411-436.

[16] A. Böcking and N. Freudenberg, Standardisierte Befunderstellung in der extragenitalen Zytologie, Der Pathologe 19 (1998), 235-258.

[17] C.J. Chen, S.C. Chang and H.H. Tseng, Assessment of immunocytochemical and histochemical stainings in the distinction between reactive mesothelial cells and adenocarcinoma cells in body effusions, Chin. Med. J. (Taipei) 54 (1994), 149155.

[18] E.S. Cibas, J.M. Corson and G.S. Pinkus, The distinction of adenocarcinoma from malignant mesothelioma in cell blocks of effusions: The role of routine mucin histochemistry and immunohistochemical assessment of carcinoembryonic antigen, keratin proteins, epithelial membrane antigen, and milk fat globule-derived antigen, Human Pathol. 18(1) 1987, 67-74

[19] A.M. Croonen, P. Van der Valk, C.J. Herman and J. Lindeman, Cytology, immunopathology and flow cytometry in the diagnosis of pleural and peritoneal effusions, Lab. Invest. 58 (1988), 725-732.

[20] V.M.J.I. Cuijpers, O.C. Boerman, M.R.J. Salet van de Pol, G.P. Vooijs, L.G. Poels and F.C.S. Ramaekers, Immunocytochemical detection of ovarian carcinoma cells in serous effusions, Acta. Cytol. 37(3) (1993), 272-279.

[21] P. Dalquen, G. Sauter, R. Epper, B. Kleiber, G. Feichter and F. Gudat, Immunocytochemistry in diagnostic cytology, in Current Status of Diagnostic Cytology, Recent Results in Cancer Research, P. Pfitzer and E. Grundmann, eds, Springer Verlag, Berlin, Heidelberg, 1993, pp. 47-80.

[22] G. Daste, G. Serre, M.A. Mauduyt, C. Vincent, P. Caveriviere and J.P. Soleilhavoup, Immunophenotyping of mesothelial cells and carcinoma cells with monoclonal antibodies to cytokeratins, vimentin, CEA and EMA improves the cytodiagnosis of serous effusions, Cytopathology 2 (1991), 19-28.
[23] M. De Angelis, I.D. Buley, A. Heryet and W. Gray, Immunocytochemical staining of serous effusions with the monoclonal antibody Ber-EP4, Cytopathology 3 (1992), 111-117.

[24] A. Dejmek and A. Hjerpe, Immunohistochemical reactivity in mesothelioma and adenocarcinoma: a stepwise logistic regression analysis, APMIS 102 (1994), 255-264.

[25] A. Dejmek, U. Brockstedt and A. Hjerpe, Optimization of a battery using nine immunocytochemical variables for distinguishing between epithelial mesothelioma and adenocarcinoma, APMIS 105 (1997), 889-894.

[26] M. Delahaye, F. van der Ham and T.H. van der Kwast, Complementary value of five carcinoma markers for the diagnosis of malignant mesothelioma, adenocarcinoma metastasis, and reactive mesothelium in serous effusions, Diagn. Cytopathol. 17(2) (1997), 115-120.

[27] C. Doglioni, A.P. Di Tos, L. Laurino, P. Iuzzolino, C. Chiarelli, M.R. Celio and G. Viale, Calretinin: a novel immunocytochemical marker for mesothelioma, Am. J. Surg. Pathol. 20(9) (1996), 1037-1046.

[28] M.A. Duggan, C.B. Masters and F. Alexander, Immunohistochemical differentiation of malignant mesothelioma, mesothelial hyperplasia and metastatic adenocarcinoma in serous effusions, utilizing staining for carcinoembryo-nicantigen, keratin and vimentin, Acta Cytol. 31 (1987), 807-814.

[29] J.M. Esteban, S. Yokota, S. Husain and H. Battifora, Immunocytochemical profile of benign and carcinomatous effusions, Am. J. Clin. Pathol. 94(6) (1990), 698-705.

[30] A. Ferrandez-Izquierdo, S. Navarro-Fos, M. Gonzalez-Devesa, R. Gil-Benso and A. Llombart-Bosch, Immunocytochemical typification of mesothelial cells in effusions: In vivo and in vitro models, Diagn. Cytopathol. 10 (1994), 256-262.

[31] M.K. Flynn, W. Johnston and S. Bigner, Carcinoma of ovarian and other origins in effusions. Immunocytochemical study with a panel of monoclonal antibodies, J. Clin. Cytol. Pathol. 37(4) (1993), 439-447.

[32] A.K. Ghosh, K.C. Gatter, M.S. Dunnill and D.Y. Mason, Immunohistological staining of reactive mesothelium, mesothelioma, and lung carcinoma with a panel of monoclonal antibodies, J. Clin. Pathol. 40 (1987), 19-25.

[33] A. Ghosh and E.B. Butler, Immunocytological staining reactions of anticarcinoembryonic antigen, $\mathrm{Ca}$, and anti-human milk fat globule monoclonal antibodies on benign and malignant exfoliated mesothelial cells, J. Clin. Pathol. 40 (1987), 1424-1427.

[34] J. Guzman, U. Costabel, K.J. Bross, U. Wiehle, F. Grunert and H.E. Schaefer, The value of the immunoperoxidase slide assay in the diagnosis of malignant pleural effusions in breast cancer, Acta Cytol. 32(2) (1988), 188-192.

[35] J. Guzman, M. Hilgarth, K.J. Bross, A. Ross, U. Wiehle, V. Kresin, F. Grunert and S. von Kleist, Malignant ascites of serous papillary ovarian adenocarcinoma. An immunocytochemical study of the tumour cells, Acta Cytol. 32(4) (1988), 519-522.

[36] J. Guzman, K.J. Bross and U. Costabel, Malignant pleural effusions due to small cell carcinoma of the lung. An immunocytochemical cell-surface analysis of lymphocytes and tumour cells, Acta Cytol. 34(4) (1990), 497-501. 
[37] J. Guzman, Immunocytochemistry: when and why?, in: Compendium on Quality Assurance, Proficiency Testing and Workload limitations in Clinical Cytology, G.L. Wied, C.M. Keebler, D.L. Rosenthal, U. Schenck, T.M. Somrak and G.P. Vooijs, eds, Tutorials of Cytology, Chicago, 1995, pp. 197-201.

[38] G. Haroske, F. Giroud, A. Reith and A. Böcking, European Society for Analytical Cellular Pathology consensus report on diagnostic DNA image cytometry. Part I: Basic considerations and recommendations for preparation, measurements and interpretation, Analyt. Cell. Pathol. (1998) (in press).

[39] A.L. Illingworth, J.A. Young and G.D. Johnson, Immunofluorescent staining of metastatic carcinoma cells in serous fluid with carcinoembryonic antibody, epithelial membrane antibody, AUA-1 and Ber-EP4, Cytopathology 5 (1994), 270 281.

[40] W.W. Johnston, C.A. Szpak, S.C. Lottich, A. Thor and J. Schlom, Use of a monoclonal antibody (B72.3) as an immunocytochemical adjunct to diagnosis of adenocarcinoma in human effusions, Cancer Res. 45 (1985), 1894-1900

[41] M.G. Joseph, D. Banerjee, P. Harris, S. Gibson and R.G. McFadden, Multiparameter flow cytometric DNA analysis of effusions: a prospective study of 36 cases compared with routine cytology and immunohistochemistry, Modern Pathol. 8(6) (1995), 686-693.

[42] L.G. Koss, Diagnostic Cytology and its Histopathologic Bases, 4th edn, J.B. Lippincott Company, Philadelphia, 1992.

[43] V. Kuenen-Boumeester, P. Van Loenen, E.M.C.A. de Bruijn and S.C. Henzen-Logmans, Quality control of immunocytochemical staining of effusions using a standardized method of cell processing, Acta Cytol. 40 (1994), 475-479.

[44] L. Kuhlmann, K.H. Berghäuser and R. Schäffer, Distinction of mesothelioma from carcinoma in pleural effusions, Path. Res. Pract. 187 (1991), 467-473.

[45] U. Latza, G. Niedobitek, R. Schwarting, H. Nekarda and H. Stein, Ber-EP4: new monoclonal antibody which distinguishes epithelia from mesothelia, J. Clin. Pathol. 43 (1990), 213-219.

[46] S.J. Lee, J.H. Nam, M.C. Lee, C.S. Park and S.W. Juhng, Immunohistochemical panel for distinguishing between carcinoma and reactive mesothelial cells in serous effusions, Acta Cytol. 40 (1995), 631-636.

[47] T.S. Loy, A.A. Diaz-Arias and J.T. Bickel, Value of BCA-225 in the cytologic diagnosis of malignant effusions: an immunocytochemical study of 197 cases, Modern Pathol. 3(3) (1990), 294-297.

[48] B. Maguire, D. Whitaker, S. Carrello and D. Spagnole, Monoclonal antibody Ber-EP4: Its use in the differential diagnosis of malignant mesothelioma and carcinoma in cell blocks of malignant effusions and FNA specimens, Diagn. Cytopathol. 10 (1994), 130-134.

[49] R.J. Marshall, A. Herbert, S.G. Braye and D.B. Jones, Use of antibodies to carcinoembryonic antigen and human milk fat globule to distinguish carcinoma, mesothelioma, and reactive mesothelium, J. Clin. Pathol. 37 (1984), 1215-1221.

[50] M.R. Mason, C. Bedrossian and C.A. Fahey, Value of immunocytochemistry in the study of malignant effusions, Diagn. Cytopathol. 3 (1987), 215-221.
[51] K.W. Matter-Walstra and R. Kraft, Atypical cells in effusions: Diagnostic value of cell image analysis combined with immunocytochemistry, Diagn. Cytopathol. 15 (1996), 263-269.

[52] F. Mayall, A. Heryet, D. Manga and A. Kriegeskotten, P53 immunostaining is a highly specific and moderately sensitive marker of malignancy in serous fluid cytology, Cytopathology 8 (1997), 9-12.

[53] J. Mezger, R. Lamerz, H. Arnholdt, D. Huhn and D. Wilmanns, Tumormarker in der Diagnostik von Aszites- und Pleurapunktaten, Onkologie 9 (1986), 11-16.

[54] H. Moch, M. Oberholzer, P. Dalquen, W. Wegmann and F. Gudat, Diagnostic tools for differentiating between pleural mesothelioma and lung adenocarcinoma in paraffin embedded tissue: Part I: Immunohistochemical findings, Virchows Arch. 423 (1993), 19-27.

[55] H. Moch, M. Oberholzer, H. Christen, M. Buser, P. Dalquen, W. Wegmann and F. Gudat, Diagnostic tools for differentiating between pleural mesothelioma from lung adenocarcinoma in paraffin embedded tissue: Part II. Design of an expert system and ist application to the diagnosis of mesothelioma, Virchows Arch. 423 (1993), 493-496.

[56] H. Motherby, T. Marcy, M. Hecker, B. Roß, B. Nadjari, H. Auer, K.M. Müller, D. Häussinger, B.E. Strauer and A. Böcking, Static DNA cytometry as a diagnostic aid in effusion cytology. I. DNA aneuploidy for identification and differentiation of primary and secondary tumours of the serous membranes, Analyt. Quant. Cytol. Histol. 20(3) (1998), 153-161.

[57] H. Motherby, B. Nadjari, T. Remmerbach, T. Marcy, N. Pomjanskaja, W. Müller, K. Knops, D. Häussinger, B.E. Strauer and A. Böcking, Static DNA cytometry as a diagnostic aid in effusion cytology. II. DNA aneuploidy for identification of neoplastic cells in equivocal effusions, Analyt. Quant. Cytol. Histol. 20(3) (1998), 162-168.

[58] K.V. Nance and J.F. Silverman, Immunocytochemical panel for the identification of malignant cells in serous effusions, Am. J. Clin. Pathol. 95(6) (1991), 867-874.

[59] N.G. Ordonez, The immunohistochemical diagnosis of mesothelioma. Differentiation of mesothelioma and lung adenocarcinoma, Am. J. Surg. Pathol. 13(4) (1989), 276-291.

[60] T. Ruitenbeek, A.S.H. Gouw ans S. Poppema, Immunocytology of body cavity fluids, Arch. Pathol. Lab. Med. 118 (1994), 265-269.

[61] K. Sheibani, S.S. Shin, J. Kezirian and L.M. Weiss, Ber-EP4 antibody as a discriminant in the differential diagnosis of malignant mesothelioma versus adenocarcinoma, Am. J. Surg. Pathol. 15(8) (1991), 779-784.

[62] P.W. Shield, J.J. Callan and P.L. Devine, Markers for metastatic adenocarcinoma in serous effusion specimens, Diagn. $C y$ topathol. 11 (1994), 237-245.

[63] J.F. Silverman, K. Nance, B. Phillips and H.A.T. Norris, The use of immunoperoxidase panels for the cytologic diagnosis of malignancy in serous effusions, Diagn. Cytopathol. 3 (1987), $134-140$.

[64] S. Singer, M.M. Boddington and E.A. Hudson, Immunocytochemical reaction of $\mathrm{Ca} 1$ and HMFG2 monoclonal antibodies with cells from serous effusions, J. Clin. Pathol. 38 (1985), 180-184. 
[65] H.K. Singh, J.F. Silverman, L. Berns, M.G. Haddad and H. Kim Park, Significance of epithelial membrane antigen in the workup of problematic serous effusions, Diagn. Cytopathol. 13 (1995), 3-7.

[66] A.I. Spriggs and M.M. Boddington, Atlas of serous fluid cytopathology. A guide to the cells of pleural, pericardial, peritoneal and hydrocele fluids, in: Current Histopathology Series, Vol. 14, G.A. Gresham, ed., Kluwer Academic Publishers, Dordrecht, 1989.

[67] R.J. Tickman, C. Cohen, V.A. Varma, P.S. Fekete and P.B. DeRose, Distinction between carcinoma cells and mesothelial cells in serous effusions. Usefulness of immunohistochemistry, Acta Cytol. 34 (1990), 491-496.

[68] D.G. Tiniakos, R.M. Healicon, T. Hair, V. Wadehra, C.H.W. Horne and B. Angus, P53 immunostaining as a marker of ma- lignancy in cytologic preparations of body fluids, Acta Cytol. 39 (1995), 171-176.

[69] A.E. Walts, J.W. Said, I.P. Shintaku, A.F. Sassoon and S. Banks-Schlegel, Keratins of different molecular weight in exfoliated mesothelial and adenocarcinoma cells - An aid to cell identification, Am. J. Clin. Pathol. 81 (1984), 442-446.

[70] J.C. Woods, A.I. Spriggs, H. Harris and J.O'D. McGee, A new marker for human cancer cells. 3 Immunocytochemical detection of malignant cells in serous fluids with the Ca1 antibody, The Lancet 4 (1982), 512-514.

[71] J.A. Zoppi, E.M. Pellicer and A.S. Sundblad, Diagnostic value of p53 protein in the study of serous effusions, Acta Cytol. 39 (1995), 1721-1724. 


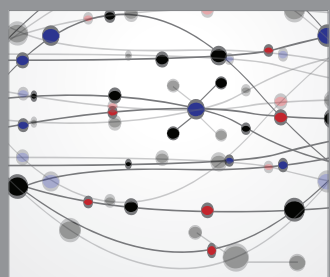

The Scientific World Journal
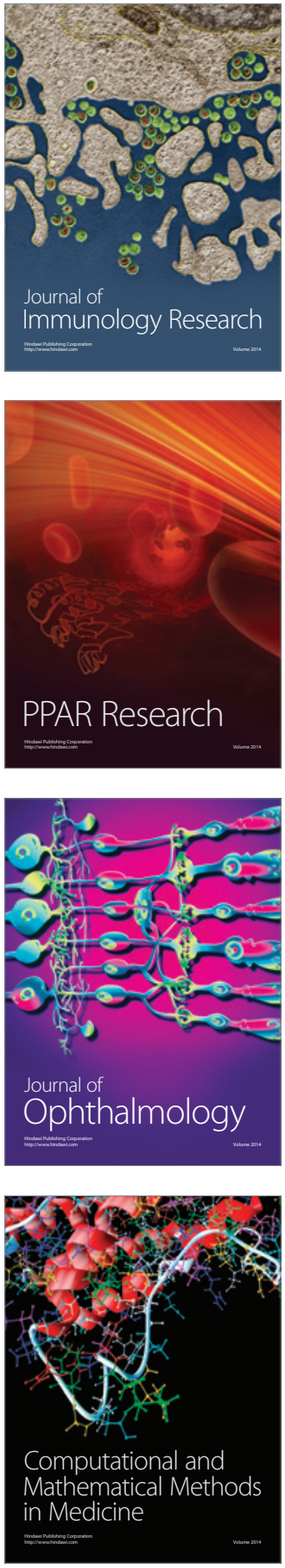

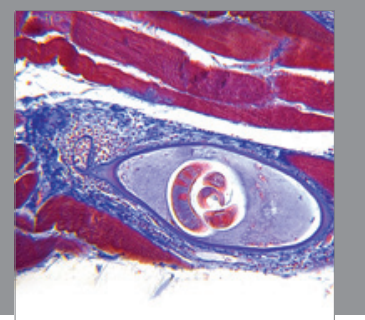

Gastroenterology

Research and Practice
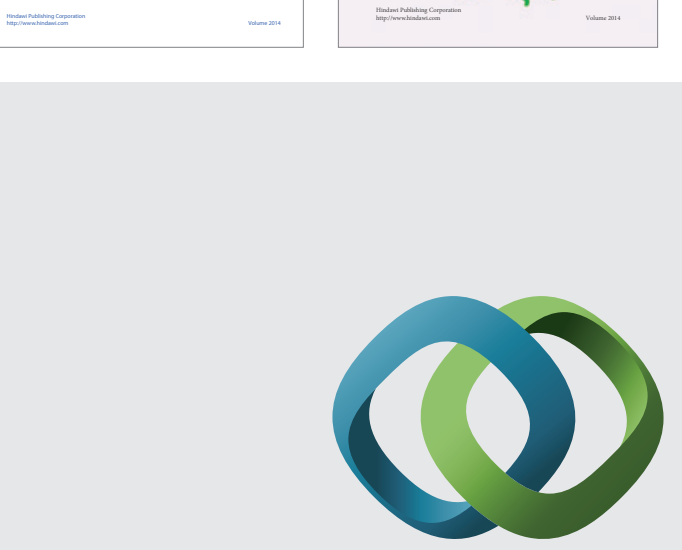

\section{Hindawi}

Submit your manuscripts at

http://www.hindawi.com
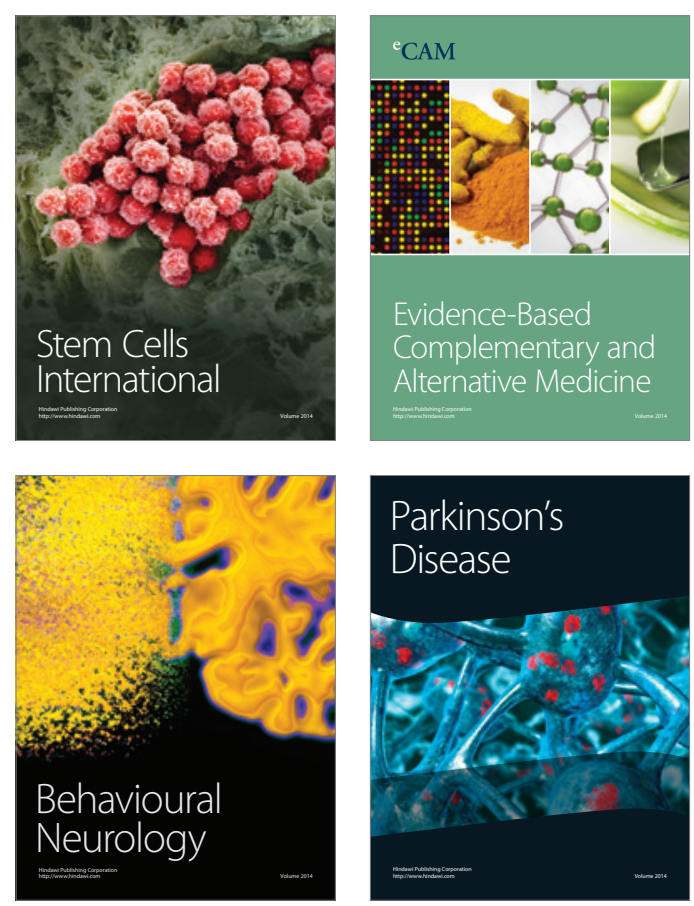

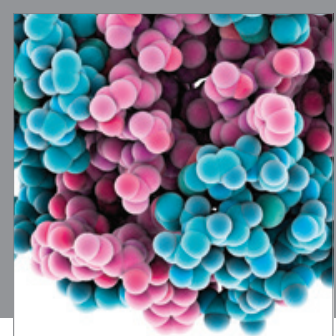

Journal of
Diabetes Research

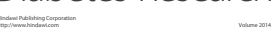

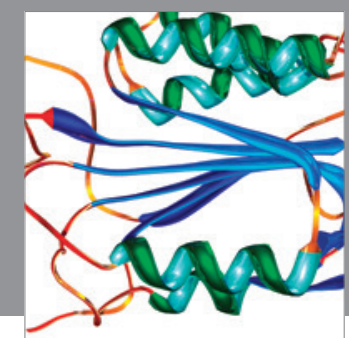

Disease Markers
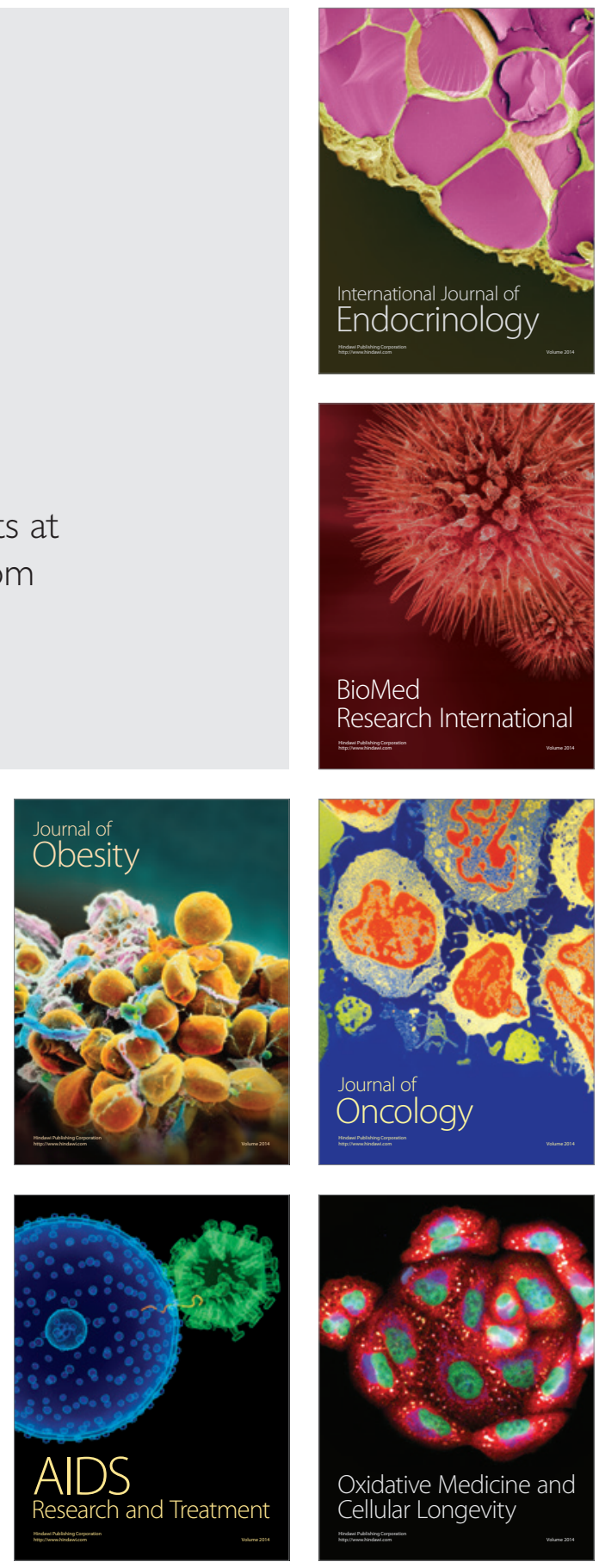